\title{
Review
}

\section{Recent advances in systemic therapy Advances in neoadjuvant (primary) systemic therapy with cytotoxic agents}

\author{
Michael Untch ${ }^{1}$ and Gunter von Minckwitz ${ }^{2}$
}

\author{
${ }^{1}$ HELIOS Klinikum Berlin-Buch, Akademisches LK der Universität Charité, Schwanebecker Chaussee 50, 13125 Berlin, Germany \\ ${ }^{2}$ German Breast Group c/o GBG Forschungs GmbH, Schleussnerstr. 42, 63263 Neu-Isenburg, Germany
}

Corresponding author: Michael Untch, michael.untch@helios-kliniken.de

Published: 17 March 2009

This article is online at http://breast-cancer-research.com/content/11/2/203

(c) 2009 BioMed Central Ltd
Breast Cancer Research 2009, 11:203 (doi:10.1186/bcr2227)

positive disease benefits from neoadjuvant chemotherapy and for whom endocrine therapy would be sufficient. Current research efforts aim to identify molecular markers at surgery which predict long-term efficacy of neoadjuvant/adjuvant endocrine treatment.

Induction of a pathologic complete response (pCR) should be one of the primary goals of neoadjuvant therapy because patients with no evidence of tumor cells in the breast or in the lymph nodes after treatment may have a longer disease-free survival (DFS) and overall survival (OS) [3]. Before any PST is started, a core biopsy should be performed to confirm the diagnosis of invasive cancer and to obtain predictive markers such as histological subtype, tumor grading according to Elston and Ellis, ER and progesterone receptor status, and HER2 status [1].

\section{Experiences from clinical studies}

A number of clinical trials have evaluated the role of neoadjuvant chemotherapy for breast cancer. One of the pioneering studies for the PST concept was the National Surgical Adjuvant Breast and Bowel Project (NSABP) B-18, which randomly assigned 1,523 women with operable breast cancer to receive four cycles of adriamycin and cyclophosphamide $(\mathrm{AC})$ either before or after definitive surgery. In the group treated with preoperative chemotherapy, a rate of $\mathrm{pCR}$, defined as the absence of residual malignant cells at the site of the primary tumor irrespective of the status of axillary nodes, of $13 \%$ was observed, with a significantly higher rate of breast conservation compared with surgery first $(67 \%$

$\mathrm{AC}=$ adriamycin and cyclophosphamide; $\mathrm{AC}-\mathrm{DOC}=$ adriamycin and cyclophosphamide with docetaxel; $\mathrm{ADOC}=$ doxorubicin and docetaxel; $\mathrm{AGO}=$ Arbeitsgemeinschaft Gynäkologische Onkologie; AT $=$ doxorubicin and paclitaxel; AT-CMF $=$ doxorubicin and paclitaxel with cyclophosphamide, methotrexate, and 5-fluorouracil; BCS = breast-conserving surgery; $c \mathrm{CR}=$ clinical complete response; $\mathrm{Cl}=$ confidence interval; $\mathrm{CMF}=$ cyclophosphamide, methotrexate, and 5-fluorouracil; DFS = disease-free survival; DOC = docetaxel; EC = epirubicin and cyclophosphamide; ER = estrogen receptor; GBG = German Breast Group; HR = hazards ratio; iv = intravenous; NSABP = National Surgical Adjuvant Breast and Bowel Project; NX = vinorelbine and capecitabine; OS = overall survival; $\mathrm{pCR}=$ pathologic complete response; PST = primary systemic therapy; TAC = docetaxel, doxorubicin, and cyclophosphamide. 
versus $60 \% ; P=0.002$ ), especially in patients with tumors larger than $5 \mathrm{~cm}$ in diameter [4].

At a median follow-up of 9 years, comparison between the groups treated with neoadjuvant and with adjuvant therapies revealed no statistically significant overall differences in either DFS or OS [5]. However, a statistically significant correlation was shown between primary tumor response and outcome: individuals achieving a pCR experienced significantly improved outcomes compared with non-pCR subjects, including 9-year DFS (75\% versus $58 \%$ ) and OS (85\% versus $73 \%)$, and a $50 \%$ decrease in the risk of death compared with all other pathologic markers (relative risk $0.50,95 \%$ confidence interval [Cl] 0.32 to 0.78 ).

In a similar study design, the European Organization for Research and Treatment of Cancer (EORTC) randomly assigned 698 women to anthracycline-based chemotherapy before or after surgery [6]. As shown in the NSABP B-18 trial, there was no significant difference in terms of OS (hazards ratio [HR] 1.16; $P=0.38$ ), progression-free survival (HR 1.15; $P=0.27$ ), or time to locoregional recurrence (HR 1.13; $P=0.61$ ) at a median follow-up of 56 months. Fiftyseven patients $(23 \%)$ were downstaged by the preoperative chemotherapy, whereas only 14 women (18\%) underwent mastectomy and not the planned breast-conserving therapy.

To examine the role of neoadjuvant taxane, the NSABP randomly assigned 2,411 women in study B-27 to neoadjuvant $A C$ alone, to neoadjuvant $A C$ followed by docetaxel before surgery, or to neoadjuvant $A C$ followed by adjuvant docetaxel after surgery. Compared with preoperative $A C$ alone, preoperative $\mathrm{AC}$ followed by docetaxel increased the clinical complete response (cCR) rate $(40.1 \%$ versus $63.6 \%$; $P<0.001)$, the overall clinical response rate $(85.5 \%$ versus $90.7 \% ; P<0.001)$, the pCR rate $(13.7 \%$ versus $26.1 \%$; $P<0.001)$, and the proportion of patients with negative nodes (50.8\% versus 58.2\%; $P<0.001$ ) [7]. Pathologic primary breast tumor response was a significant predictor of pathologic nodal status $(P<0.001)$. At a median follow-up of 6.5 years, women achieving $\mathrm{pCR}$ demonstrated significantly improved survival compared with those without pCR (HR $0.33,95 \% \mathrm{Cl} 0.23$ to $0.47 ; P<0.0001$ ) [8].

In the European Cooperative Trial in Operable breast cancer (ECTO), women with operable breast cancer (primary tumor greater than $2 \mathrm{~cm}$ ) were randomly assigned to adjuvant doxorubicin (75 mg/m² every 21 days) for four cycles followed by CMF (cyclophosphamide, methotrexate, and 5 -fluorouracil) or to adjuvant doxorubicin $\left(60 \mathrm{mg} / \mathrm{m}^{2}\right)$ and paclitaxel ( $200 \mathrm{mg} / \mathrm{m}^{2}$ over the course of 3 hours every 21 days) for four cycles followed by CMF (AT-CMF) or to AT-CMF as PST, yielding a cCR in $52 \%$ of patients $(27 \%$ after AT and $25 \%$ more after CMF) [9]. pCR was documented in $23 \%$ of specimens and was associated with negative axillary nodes in $87 \%$. Conservative surgery was more frequent after PST
(71\% versus $35 \%$ before adjuvant therapy; $P<0.00001$ ) regardless of tumor size at diagnosis. The frequency of pathologically negative nodes was also significantly higher in the PST group (61\% versus $38 \%$ ).

During the same meeting, the results of the German Gynecological Oncology Working Group (Arbeitsgemeinschaft Gynäkologische Onkologie, or AGO) study were also presented, demonstrating the feasibility of a dose-dense biweekly protocol [10]. This trial was designed to compare the frequency of BCS, the response rates, and the safety between two epirubicin- and paclitaxel-containing regimens given either as dose-dense sequential intensified chemotherapy ( $\operatorname{arm} A)$ or in a standard dose (arm B), both as preoperative therapy for primary breast cancer. Patients with large primary tumors $(>3 \mathrm{~cm})$ or inflammatory disease were randomly assigned to receive either three cycles of epirubicin $150 \mathrm{mg} / \mathrm{m}^{2}$ followed by three cycles of paclitaxel $250 \mathrm{mg} / \mathrm{m}^{2}$ every 2 weeks with granulocyte colony-stimulating factor support or four cycles of combination epirubicin $90 \mathrm{mg} / \mathrm{m}^{2}$ and paclitaxel $175 \mathrm{mg} / \mathrm{m}^{2}$ every 3 weeks as preoperative therapy. A total of 631 patients were enrolled. Preliminary data from 475 patients demonstrated a significantly higher frequency of BCS $(66 \%$ versus $55 \% ; P=0.016)$, pCR (18\% versus $10 \% ; P=0.03)$, and negative axillary lymph nodes at surgery $(51 \%$ versus $42 \% ; P=0.098$ ) with the every-2-week regimen.

The phase III GeparDuo (the second German Preoperative Adriamycin and Docetaxel (GerparDo) trial) $(n=913)$ of the German Adjuvant Breast Cancer Group (GABG) compared the pathologic locoregional complete response rate achieved with preoperative administration of the 8-week dose-dense combination regimen ADOC (doxorubicin $50 \mathrm{mg} / \mathrm{m}^{2}$ plus docetaxel $75 \mathrm{mg} / \mathrm{m}^{2}$ every 14 days for four cycles with filgrastim support), as studied in the predecessor study GeparDo, with that of a 24-week sequential schedule of $A C$ followed by docetaxel (AC-DOC) (doxorubicin $60 \mathrm{mg} / \mathrm{m}^{2}$ plus cyclophosphamide $600 \mathrm{mg} / \mathrm{m}^{2}$ every 21 days followed by docetaxel $100 \mathrm{mg} / \mathrm{m}^{2}$ every 21 days for four cycles each), similar to one of the treatment arms in the NSABP B-27 trial [11].

A pCR was achieved in 94 patients (10.6\%), but the likelihood was significantly greater with AC-DOC (14.3\%; $n=63)$ than with the ADOC regime $(7.0 \% ; n=31)$ (odds ratio $2.22,90 \% \mathrm{Cl} 1.52$ to $3.24 ; P<0.001$ ). Independent predictors of achieving a pCR included the use of sequential therapy, high tumor grade, and negative hormone receptor status. The response rates detected by palpation and by imaging were significantly higher with AC-DOC (85.0\% and $78.6 \%$, respectively) than with ADOC (75.2\% and $68.6 \%$, respectively; both $P$ values $<0.001)$. The rates of BCS were 63.4\% for AC-DOC and 58.1\% for ADOC $(P=0.05)$.

\section{Evaluation of early response}

As shown in the studies, women with an early or mid-course response to neoadjuvant chemotherapy have chemosensitive 
tumors and a high probability for a pCR at surgery. Therefore, assessing early tumor response to chemotherapy is crucial to avoid unnecessary toxicity without potential benefit from the treatment. Neoadjuvant chemotherapy response is currently achieved by monitoring changes in tumor size using clinical examination based on palpable change in tumor size backed up by mammography and/or ultrasound.

Relevant changes (for example, partial remissions, defined as a reduction in the product of the two largest perpendicular diameters of the primary tumor size by $50 \%$ or more) in tumor size can be observed at as early as 4 to 6 weeks (that is, two cycles) of chemotherapy. Early detection of response might therefore be used as follows [2]:

- as a predictor of pathologic response

- as a predictor of long-term outcome

- as a decision aid to switch therapy

- to identify patients who might or might not benefit from a switch in therapy.

One approach to use this early information on response in the clinical setting to tailor further treatment strategies for individual patient therapy has been evaluated in the GeparTrio trial, the first prospective randomized study to address patients with tumors that do or do not show an early response [12]. Of 2,090 patients enrolled in the GeparTrio study, 622 (29.8\%) who did not respond to two initial cycles of TAC (docetaxel at $75 \mathrm{mg} / \mathrm{m}^{2}$, doxorubicin at $50 \mathrm{mg} / \mathrm{m}^{2}$, and cyclophosphamide at $500 \mathrm{mg} / \mathrm{m}^{2}$ ) with a decrease in tumor size by at least $50 \%$ were randomly assigned to switch to a better tolerated non-cross-resistant regimen consisting of four additional cycles of TAC $(n=321)$ or to four cycles of vinorelbine at $25 \mathrm{mg} / \mathrm{m}^{2}$ and capecitabine at $2,000 \mathrm{mg} / \mathrm{m}^{2}$ (NX) $(n=301)$.

Sonographic response rates were $50.5 \%$ for the TAC arm and $51.2 \%$ for the NX arm. The difference of $0.7 \%(95 \% \mathrm{Cl}$ $-7.1 \%$ to $8.5 \%)$ demonstrated non-inferiority of NX $(P=0.008)$. Similar numbers of patients in the two arms received BCS (184 [57.3\%] in the TAC arm versus 180 [59.8\%] in the NX arm) and had a pCR (5.3\% versus $6.0 \%)$. Fewer patients in the NX arm than in the TAC group had hematological toxic effects, mucositis, infections, and nail changes, but more had hand-foot syndrome and sensory neuropathy. In conclusion, pCRs to both regimens were marginal. Among patients who did not respond to the initial neoadjuvant TAC treatment, similar efficacy but better tolerability was observed by switching to NX than continuing with TAC.

The GeparTrio study also examined the benefit of an intensified neoadjuvant chemotherapy regime consisting of an additional four $(n=704)$ or six $(n=686)$ TAC cycles for those women who responded to two initial cycles of TAC $(n=1,390)$ [13]. Patients receiving a total of eight TAC cycles had statistically significantly higher sonographic response rates, but not $\mathrm{pCR}$ rates, than those receiving six TAC cycles. However, eight TAC cycles showed more side effects. Therefore, eight cycles of TAC cannot be recommended for the whole group of patients responding to two initial cycles of TAC.

\section{Predictors of response}

Efforts have been made to identify more accurately the likelihood of pCR under neoadjuvant chemotherapy. The most important predictive marker concerning response to a preoperative taxane-anthracycline-based regimen is negative hormone receptor status. However, despite a pathologic complete remission rate exceeding $40 \%$, survival of patients with this phenotype was reported in several studies to be shorter than for those with receptor-positive tumors [1]. Some studies identified a lower response rate for operable invasive lobular carcinomas. So far, trials with various biological markers like HER2 and topoisomerase lla have revealed heterogeneous data concerning the prediction of response to specific therapies. Currently, a set of biological markers, rather than a single one, seem to be important to differentiate between a high or low chance for a pCR.

\section{New primary systemic therapy concepts}

Patients with no pCR have a significant risk of recurrent disease, and currently no further standard therapy exists. Therefore, alternative regimens are urgently needed to improve therapeutic outcomes for this high-risk population. Ongoing or not-yet-published neoadjuvant trials integrate modern concepts of treatment like tumor targeting with new biological agents or bisphosphonates.

The phase III GeparQuattro study conducted by the AGO and German Breast Group (GBG) study groups is the largest neoadjuvant clinical trial in women with HER2-positive breast cancer. One thousand five hundred and ten women received four cycles of EC (epirubicin $90 \mathrm{mg} / \mathrm{m}^{2}$ and cyclophosphamide $600 \mathrm{mg} / \mathrm{m}^{2}$ ) and were randomly assigned to either four cycles of docetaxel $\left(100 \mathrm{mg} / \mathrm{m}^{2}\right)$ (arm A) or four cycles of docetaxel $\left(75 \mathrm{mg} / \mathrm{m}^{2}\right)$ plus capecitabine $\left(1,800 \mathrm{mg} / \mathrm{m}^{2}\right)$ (arm B) or four cycles of docetaxel $\left(75 \mathrm{mg} / \mathrm{m}^{2}\right)$ followed by four cycles of capecitabine $\left(1,800 \mathrm{mg} / \mathrm{m}^{2}\right)$ (arm C) (Figure 1). Women with HER2-positive tumors $(n=456)$ received trastuzumab 6 (8) $\mathrm{mg} / \mathrm{kg}$ of body weight every 3 weeks concomitantly with all neoadjuvant chemotherapy before surgery and for up to 1 year after surgery. To minimize the cardiac risk, patients with any previous heart problems and/or an ejection fraction below 55\% were excluded. As the analysis of efficacy has shown, the pCR rate (primary endpoint) in women with HER2-positive tumors was significantly increased by the addition of trastuzumab $(45.5 \%$ versus $19.5 \%$ ) (Figure 2). The addition of trastuzumab to the 


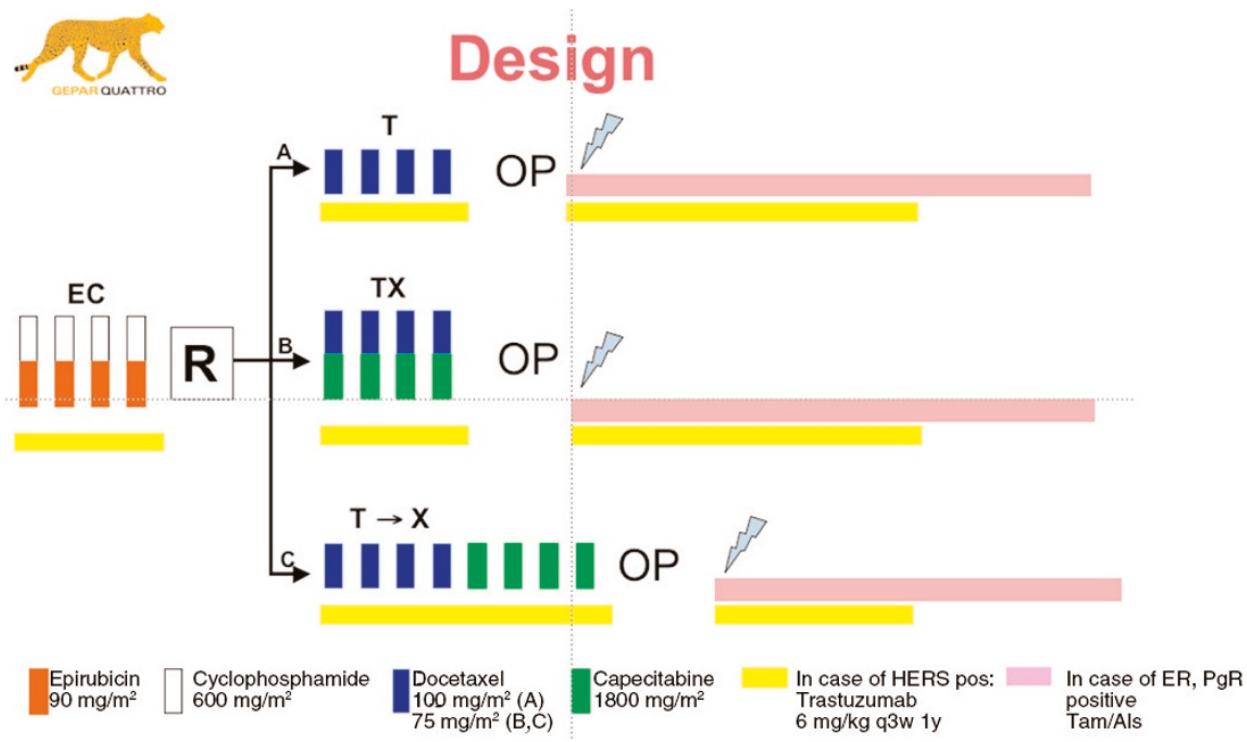

Study design of the GeparQuattro study. EC, epirubicin and cyclophosphamide; ER, estrogen receptor; OP, operation (surgery); PR, partial response; R, random assignment; T, trastuzumab; X, capecitabine. Reprinted with permission [17].

combination of EC and docetaxel and capecitabine is feasible without clinically relevant cardiotoxicity [14] (Figure 3). The addition of capecitabine, neither concomitantly nor in sequence, did not improve the pCR rate.

The multicenter phase II TECHNO (Taxol-Epirubicin-Cyclophosphamide-Herceptin Neoadjuvant) study of the AGO evaluates preoperative $4 \times \mathrm{EC} 90 / 600 \mathrm{mg} / \mathrm{m}^{2}$ every 3 weeks followed by $4 \times$ paclitaxel $175 \mathrm{mg} / \mathrm{m}^{2}$ every 3 weeks with a trastuzumab loading dose of $8 \mathrm{mg} / \mathrm{kg}$ followed by $6 \mathrm{mg} / \mathrm{kg}$ every 3 weeks, followed by surgery and postoperative trastuzumab $6 \mathrm{mg} / \mathrm{kg}$ every 3 weeks for 9 months in 230 patients with HER2-positive breast cancer (immunohistochemistry $3+$ or fluorescence in situ hybridization [FISH]positive confirmed by central pathology) [15]. Radiotherapy and endocrine therapy were applied according to standard recommendations. In 119 analyzed patients, 37\% achieved a histopathologic complete response and $17 \%$ had only residual ductal carcinoma in situ in the breast. Seventy-three percent of women showed histologically negative axillary nodes at surgery. Updated data were scheduled to be available at the end of last year.

The currently recruiting, randomized, open-label multicenter phase III Neo-ALTTO (Neoadjuvant Lapatinib and/or Trastuzumab Treatment Optimisation) study is comparing the efficacy of neoadjuvant lapatinib (a novel orally active smallmolecule and dual-tyrosine kinase inhibitor of both epidermal growth factor receptor and HER2) plus paclitaxel, versus trastuzumab plus paclitaxel, versus concomitant lapatinib and trastuzumab plus paclitaxel given as neoadjuvant treatment in
HER2-overexpressing and/or amplified primary breast cancer (Figure 4). Patients will be randomly assigned to receive lapatinib $1,500 \mathrm{mg}$ daily, trastuzumab $4 \mathrm{mg} / \mathrm{kg}$ intravenous (iv) load followed by $2 \mathrm{mg} / \mathrm{kg}$ iv weekly, or the lapatinib $1,000 \mathrm{mg}$ daily with trastuzumab $4 \mathrm{mg} / \mathrm{kg}$ iv load followed by $2 \mathrm{mg} / \mathrm{kg}$ iv weekly for a total of 6 weeks. After this biological window, patients will continue on the same targeted therapy plus weekly paclitaxel $80 \mathrm{mg} / \mathrm{m}^{2}$ for a further 12 weeks, up to definitive surgery. After surgery, patients will receive three courses of adjuvant chemotherapy with FEC followed by the same targeted therapy as in the neoadjuvant setting for a further 34 weeks. The planned total duration of the anti-HER2 therapy will be 1 year. The primary objective of this study is to evaluate and compare the rate of $\mathrm{pCR}$ at the time of surgery (18 weeks) in patients randomly assigned to receive neoadjuvant lapatinib or trastuzumab or their combination plus paclitaxel. The estimated enrollment will be 450 patients. The study started in November 2007 and will be completed in September 2009.

The PREPARE (Preoperative Epirubicin Paclitaxel Aranesp) phase III study was initiated in 2002 and was developed, conducted, and analyzed by the AGO and GBG. It was designed to evaluate the relapse-free survival time and $O S$ of a sequential dose-dense and dose-intensified regimen of epirubicin, paclitaxel, and CMF compared with preoperative sequential administration of epirubicin and cyclophosphamide followed by paclitaxel sequential interval-shortened and dose-intensified preoperative use of epirubicin, paclitaxel, and CMF with preoperative sequential administration of epirubicin and cyclophosphamide followed by paclitaxel in 


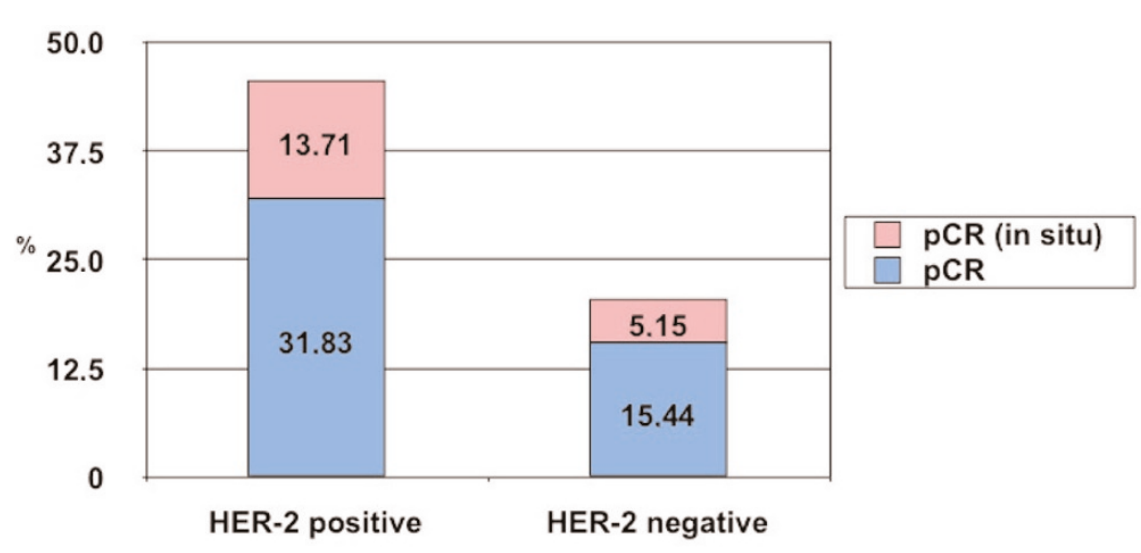

GeparQuattro study: pathologic complete response (pCR) rates in women with HER2-positive or -negative breast cancer.

\section{Figure 3}

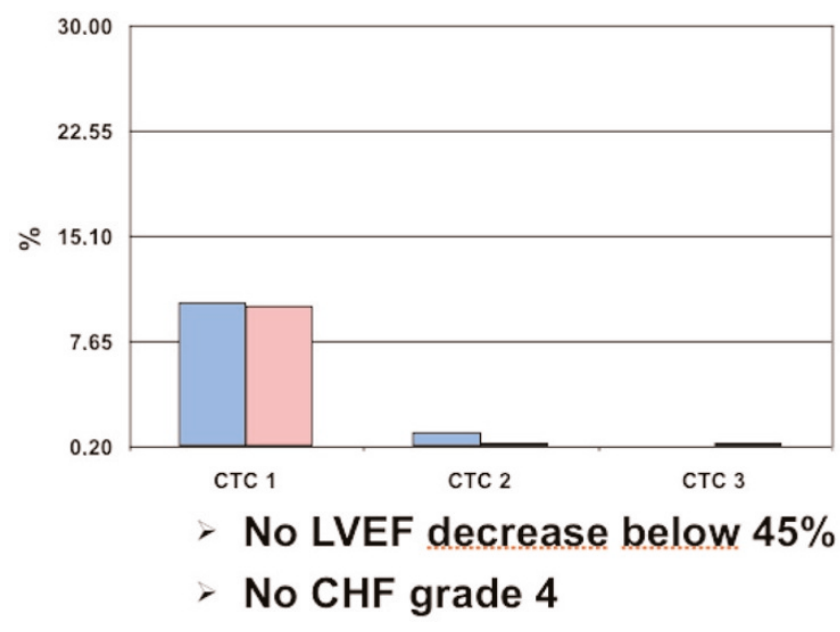

HER-2 negative / no trastuzumab

HER-2 positive I with trastuzumab

GeparQuattro study: cardiac events in women with HER2-positive and -negative breast cancer. CHF, congestive heart failure; CTC, crackle transmission coefficient; LVEF, left ventricular ejection fraction.

733 women with primary breast cancer. Pegfilgrastim was used as a secondary preventive after febrile neutropenia in the standard arm of the study or, in exceptional cases, after severe febrile neutropenia necessitating the postponement of treatment by more than 1 week. In addition, the influence of darbepoetin alfa on response rate and quality of life was investigated in both treatment arms. An interim analysis showed that the $\mathrm{pCR}$ rate was significantly higher in the dose-intensified arm compared with the standard arm (18.7\% versus $13.2 \% ; P=0.0425)$. The use of darbepoetin alfa to support neoadjuvant chemotherapy kept hemoglobin levels stable and had no significant impact on tumor response to chemotherapy at the time of surgery [16]. Long-term followup data showed no differences with regard to the comparison of the two chemotherapy regimen. However, there were more relapses and deaths in the group of patients treated with darbepoetin alpha.

The current study of the GBG and AGO groups, GeparQuinto, is addressing three questions with randomizations for separate subgroups: (1) In patients with HER2-negative tumors, we investigate the role of bevacizumab given concomitantly to epirubicin/cyclophosphamide (EC) followed by docetaxel (D) chemotherapy. (2) In patients with HER2negative tumors not responding to $E C$ with or without bevacizumab, we investigate the role of Everolimus (RAD 001) in combination with paclitaxel. (3) In patients with HER2positive tumors we compare lapatinib against trastuzumab 


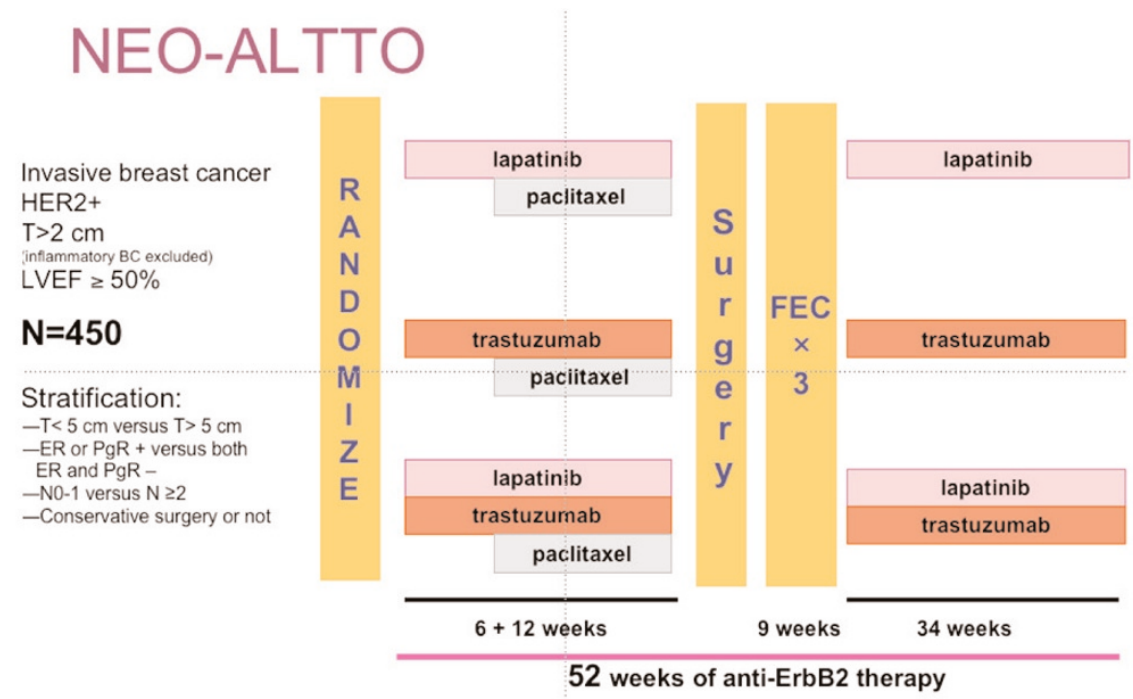

Design of the Neoadjuvant Lapatinib and/or Trastuzumab Treatment Optimisation (Neo-ALTTO) study. ER, estrogen receptor; FEC, fluorouracil, epirubicin, and cyclophosphamide; LVEF, left ventricular ejection fraction; PgR, progesterone receptor; T, trastuzumab.

given concomitantly with EC-D. As of March 2009, the trials have recruited 600 out of 2400 patients.

\section{Conclusions}

Neoadjuvant chemotherapy for early breast cancer is a major development with important implications for the management of this disease. Originally used only in women with locally advanced inoperable breast cancer, PST can now be offered as a very good option for primary operable disease in patients who are candidates for adjuvant systemic chemotherapy, irrespective of the size of the tumor [1].

The available data suggest a significant and important correlation between pCR after neoadjuvant therapy and DFS as well as OS. Moreover, PST increases the rate of BCSs and is associated with a lower rate of positive axillary lymph nodes at the time of surgery [1]. The preoperative addition of a taxane to preoperative $A C$ results in a significant increase in the rate of $c C R, p C R$, and negative axillary nodes in patients with operable breast cancer.

At least six cycles of an anthracycline- and taxane-containing regimen should be planned and given preoperatively over the

This article is part of a review series on

Recent advances in systemic therapy, edited by Paul Ellis.

Other articles in the series can be found online at http://breast-cancer-research.com/series/ bcr_systemic therapy course of 4 to 6 months [1]. Trastuzumab is recommended for patients with HER2-positive tumors. The concurrent use of the anti-HER2 antibody with an anthracycline-containing regimen should be given only in clinical trials. Individualization of neoadjuvant therapy for breast cancer according to midcourse response or to molecular tumor characteristics will be one of the most important goals in the coming years.

\section{Competing interests}

GvM has received research funding from Sanofi-Aventis, Roche, Amgen, BMS, GSK and Novartis. MU has received research funding from Amgen, BMS and GSK.

\section{References}

1. Kaufmann M, von Minckwitz G, Bear HD, Buzdar A, McGale P, Bonnefoi $H$, Colleoni M, Denkert C, Eiermann W, Jackesz R, Makris A, Miller W, Pierga JY, Semiglazov V, Schneeweiss A, Souchon R, Stearns V, Untch M, Loibl S: Recommendations from an international expert panel on the use of neoadjuvant (primary) systemic treatment of operable breast cancer: new perspectives 2006. Ann Oncol 2007, 18:1927-1930.

2. von Minckwitz G: Can we tailor chemotherapy based on preliminary response? General session X: multidisciplinary implications of preoperative therapy. Paper presented at: American Society of Clinical Oncology 2008 Breast Cancer Symposium; 57 September 2008; Washington, DC.

3. Bonadonna G, Valagussa P, Brambilla C, Ferrari L, Moliterni A, Terenziani $M$, Zambetti M: Primary chemotherapy in operable breast cancer: eight-year experience at the Milan Cancer Institute. J Clin Oncol 1998, 16:93-100.

4. Fisher B, Bryant J, Wolmark N, Mamounas E, Brown A, Fisher ER, Wickerham DL, Begovic M, DeCillis A, Robidoux A, Margolese RG, Cruz AB Jr., Hoehn JL, Lees AW, Dimitrov NV, Bear HD: Effect of preoperative chemotherapy on the outcome of women with operable breast cancer. J Clin Oncol 1998, 16: 2672-2685.

5. Wolmark N, Wang J, Mamounas E, Bryant J, Fisher B: Preoperative chemotherapy in patients with operable breast cancer: nine-year results from National Surgical Adjuvant Breast and 
Bowel Project B-18. J Natl Cancer Inst Monogr 2001, (30):96102.

6. Van der Hage JA, van de Velde CJ, Julien JP, Tubiana-Hulin M, Vandervelden C, Duchateau L: Preoperative chemotherapy in primary operable breast cancer: results from the European Organization for Research and Treatment of Cancer trial 10902. J Clin Oncol 2001, 19:4224-4237.

7. Bear HD, Anderson S, Brown A, Smith R, Mamounas EP, Fisher B, Margolese R, Theoret H, Soran A, Wickerham DL, Wolmark N; National Surgical Adjuvant Breast and Bowel Project Protocol B27: The effect on tumor response of adding sequential preoperative docetaxel to preoperative doxorubicin and cyclophosphamide: preliminary results from National Surgical Adjuvant Breast and Bowel Project Protocol B-27. J Clin Oncol 2003, 21:4165-4174.

8. Bear HD, Anderson S, Smith RE, Geyer CE Jr., Mamounas EP, Fisher B, Brown AM, Robidoux A, Margolese R, Kahlenberg MS, Paik S, Soran A, Wickerham DL, Wolmark N: Sequential preoperative or postoperative docetaxel added to preoperative doxorubicin plus cyclophosphamide for operable breast cancer: National Surgical Adjuvant Breast and Bowel Project Protocol B-27. J Clin Oncol 2006, 24:2019-2027.

9. Gianni L, Baselga J, Eiermann W, Porta VG, Semiglazov V, Garcia-Conde J, Zambetti M, Valagussa P, Bonadonna G, for the ECTO Study Group: First report of the European Cooperative Trial in operable breast cancer (ECTO): effects of primary systemic therapy (PST) on local-regional disease. Proc Am Soc Clin Oncol 2002, 21:34a. Abstract 132.

10. Untch M, Möbus V, Kuhn W, Muck BR, Thomssen C, Bauerfeind I, Harbeck N, Werner C, Lebeau A, Schneeweiss A, Kahlert S, von Koch F, Petry KU, Wallwiener D, Kreienberg R, Albert US, Lück $H J$, Hinke A, Jänicke $F$, Konecny GE: Intensive dose-dense compared with conventionally scheduled preoperative chemotherapy for high-risk primary breast cancer. J Clinical Oncol 2009, in press.

11. Von Minckwitz G, Raab G, Caputo A, Schütte M, Hilfrich J, Blohmer JU, Gerber B, Costa SD, Merkle E, Eidtmann H, Lampe D, Jackisch C, du Bois A, Kaufmann M: Doxorubicin with cyclophosphamide followed by docetaxel every 21 days compared with doxorubicin and docetaxel every 14 days as preoperative treatment in operable breast cancer: the GEPARDUO study of the German Breast Group. J Clin Oncol 2005, 23:2676-2678.

12. Von Minckwitz $G$, Kümmel $S$, Vogel $P$, Hanusch $C$, Eidtmann $H$, Hilfrich J, Gerber B, Huober J, Costa SD, Jackisch C, Loibl S, Mehta K, Kaufmann M; German Breast Group: Neoadjuvant vinorelbine-capecitabine versus docetaxel-doxorubicincyclophosphamide in early nonresponsive breast cancer: phase III randomized GeparTrio trial. J Natl Cancer Inst 2008, 100:542-551.

13. von Minckwitz G, Kümmel $S$, Vogel $P$, Hanusch $C$, Eidtmann $H$, Hilfrich J, Gerber B, Huober J, Costa SD, Jackisch C, Loibl S, Mehta K, Kaufmann M; German Breast Group: Intensified neoadjuvant chemotherapy in early-responding breast cancer: phase III randomized GeparTrio study. J Natl Cancer Inst 2008, 100:552-562.

14. Untch M: Neoadjuvant treatment of HER2 overexpressing primary breast cancer with trastuzumab given concomitantly to epirubicin/cyclophosphamide followed by docetaxel \pm capecitabine. First analysis of efficacy and safety of the GBG/AGO multicenter intergroup-study "GeparQuattro". Paper presented at: 6th European Breast Cancer Conference; 15-19 April 2008; Berlin, Germany. Abstract 0450.

15. Untch $M$, Stoeckl D, Konecny $G$, Kreienberg $R$, Thommsen $C$ Camara O, Moebus V, Kuehn T, DuBois A, Lueck HJ, Gitsch G, Fasching P: A multicenter phase II study of preoperative epirubicin, cyclophosphamide (EC) followed by paclitaxel $(P)$ plus trastuzumab $(\mathrm{T})$ in Her2 positive primary breast cancer. Paper presented at: 28th Annual San Antonio Breast Cancer Symposium; 8-11 December 2005; San Antonio, TX. Abstract 1064.

16. Untch $M$, Fasching PA, Stöckl D, Kahlert $S$, Conrad U, Camara $O$ Fett W, Kurzeder C, Lück HJ, Loibl S, von Minckwitz G: PREPARE trial. A randomized phase III trial comparing preoperative, dose dense, dose-intensified chemotherapy with epirubicin, paclitaxel and CMF with standard dosed epirubicin/ cyclophosphamide followed by paclitaxel \pm darbepoetin alfa in primary breast cancer: a preplanned interim analysis of efficacy at surgery. ASCO 2008. J Clin Oncol 2008, 26 (suppl): abstract 517.

17. von Minckwitz G, Rezai M, Loibl S, Fasching P, Huober J, Tesch $H$, Bauer-feind I, Hilfrich J, Mehta K, Untch M: Evaluating the efficacy of capecitabine given concomitantly or in sequence to epirubicin/cyclophosphamide docetaxel as neoadjuvant treatment for primary breast cancer. First efficacy analysis of the GBG/AGO intergroup-study GeparQuattro. Paper presented at: 30th Annual San Antonio Breast Cancer Symposium; 13-16 December 2007; San Antonio, TX. Abstract 79. 